\title{
An Oosterhoff Analysis of the Galactic Bulge Field RR Lyrae stars: Implications On Their Absolute Magnitudes
}

\author{
Andrea Kunder and Brian Chaboyer \\ Dartmouth College, 6127 Wilder Lab, Hanover, NH 03755 \\ E-mail: Andrea.M.Kunder@Dartmouth.edu and Brian.Chaboyer@dartmouth.edu
}

\begin{abstract}
We present an analysis of the period- $V$-amplitude plane for RR0 Lyrae stars (fundamental mode pulsators) with "normal" light curves in the bulge using the MACHO bulge fields. Although bulge globular clusters have RR Lyraes that divide into two reasonable distinct groups according to the average period of the RR0 Lyraes (Oosterhoff 1939), there is no evidence of a gap between Oosterhoff I and II stars in the bulge field star sample. The majority of the bulge RR0 Lyrae field star population have a difference in period compared to the Oosterhoff I cluster M3 $(\Delta \log \mathrm{P})$ that is shifted by about 0.02 days with regard to the Milky Way Oosterhoff I population, and the sample includes stars with $\Delta \log \mathrm{P}>0.06$ days, a characteristic hardly seen in Milky Way globular clusters. The metal-rich RR0 Lyrae stars in the Galactic bulge sample have $\Delta \log \mathrm{P}$ values on the other side of the spectrum as those in the metal-rich globular clusters NGC 6388 and NGC 6441 . We find that the $V$-amplitude for a given period is a function of $\Delta \log \mathrm{P}$, and not of metal abundance, similar to the result found by Clement \& Shelton (1999) for RR Lyrae stars in Milky Way globular clusters. A comparative study of the bulge field stars with similar metallicities but different Oosterhoff types is carried out. Bulge field RR0 Lyrae variables with $\Delta \log \mathrm{P}$ values similar to Oosterhoff II clusters are about 0.2 mag brighter than RR0 Lyrae variables with $\Delta \log \mathrm{P}$ similar to Oosterhoff I clusters. Reliance upon a single $\mathrm{M}_{\mathrm{V}}^{\mathrm{RR}}-[\mathrm{Fe} / \mathrm{H}]$ relation may be inappropriate when considering populations with different $\Delta \log \mathrm{P}$.
\end{abstract}

Subject headings: surveys - stars: abundances, distances, Population II — Galaxy: center

\section{Introduction}

RR Lyrae stars are the most popular distance indicators for old, low mass stars (see, e.g., Carretta et al. 2000; Walker 2000). The knowledge of their absolute magnitude and its possible dependence on metallicity allows the determination of basic astronomical parameters such as distance to the galactic center and Local Group galaxies, as well as distances and ages of the Milky 
Way globular clusters. These have a connection with the Hubble constant and with the conditions of the Milky Way's Galaxy formation.

It was shown by Oosterhoff (1939) that globular clusters (GCs) divide into two groups, based on the pulsational properties of their fundamental mode pulsating RR0 Lyrae stars. Later it was found that these groups could also be separated by metallicity. The Oosterhoff I (OoI) type GCs have an average RR0 Lyrae pulsational period of 0.55 days and metallicities that are more metal rich than -1.5 dex. The Oosterhoff II (OoII) type GCs have an average RR0 Lyrae pulsational period of 0.65 days and are more metal poor than -1.5 dex. There is a gap at the two period groups separated by a mean period ratio of $\log 0.65 / 0.55=0.073$. This is now termed the Oosterhoff dichotomy. No physical reason for this dichotomy in periods is known to date, but a difference in mean luminosity between the two groups of about 0.2 mag could explain the observations (Sandage 2006). Temperature differences alone between the two Oosterhoff period groups are not sufficient to account for this luminosity difference (Sandage 1958).

It has long been customary to express the luminosities of RR Lyrae variables by

$$
M_{V}=a+[\mathrm{Fe} / \mathrm{H}] b .
$$

However, there has been much dispute as to the values of $a$ and $b$. The question of the linearity of the equation is also now being investigated. The evidence for non-linearity is from (a) theoretical models of the horizontal-branch (HB) (both at zero age and in an evolved state), (b) the pulsation equation using the observed input parameters of mass- $[\mathrm{Fe} / \mathrm{H}]$ and $\log \mathrm{T}_{\mathrm{e}}$ (from colors) as functions of metallicity relations, (c) and from semiempirical use of observational data (Sandage \& Tammann 2006). For example, Bono, Caputo, \& Di Criscienzo (2007) use RR Lyrae in the Milky Way show the nonlinearity (see their Fig. 16) over the entire range of $[\mathrm{Fe} / \mathrm{H}]$ from 0 to -2.5 dex.

The derived zero points of the nonlinear $M_{V}^{\mathrm{RR}}-[\mathrm{Fe} / \mathrm{H}]$ relation depends on the investigations and methods used. Some lead to the so-called long RR Lyrae scale that gives $\left\langle M_{V}\right\rangle \sim+0.52$ at $[\mathrm{Fe} / \mathrm{H}]=-1.5$ dex and some lead to the short scale that gives $\left\langle M_{V}\right\rangle \sim+0.72$ at $[\mathrm{Fe} / \mathrm{H}]=-1.5$ dex. Gaining an understanding of the Oosterhoff dichotomy will likely aid in understanding the absolute magnitude calibration of RR Lyrae stars. In this paper we look at the Oosterhoff properties of bulge field stars and present evidence that even nonlinear $[\mathrm{Fe} / \mathrm{H}]$ relations cannot fully account for finding their absolute magnitudes.

Adding to the Oosterhoff puzzle, Local Group Dwarf spheroidal galaxies (dSph) and LMC globular clusters have RR0 Lyrae variables with properties that fill the gap in a plot of $[\mathrm{Fe} / \mathrm{H}]$ versus fundamental mode period as illustrated by Siegel et al. (2000), Pritzl (2004) and Catelan (2005). Studying the Oosterhoff properties of GCs in both our Galaxy and those belonging to the dwarf companions of the Milky Way aid in identifying the building blocks of the Galactic halo. Identifying the origin of the Oosterhoff dichotomy is hence helpful in exploring the formation of the Milky Way galaxy. For example, if the Searle \& Zinn (1978) Galaxy formation scenario (hierarchical merging and continual accretion of lower mass protogalactic fragment formation scenario) is dominant, then one would imagine that the RR Lyrae variables in the Local Group dSph's and the RR Lyrae 
variables in the LMC globular clusters would have similar properties to that of the Milky Way RR Lyrae stars (see, e.g., Catelan 2007).

One explanation for the Oosterhoff dichotomy was put forth by van Albada \& Baker (1971), They suggested that the evolutionary scenario of RR Lyrae in OoI and OoII clusters differs. The horizontal-branch (HB) stars in the intermediate metallicity OoI clusters begin their lives near the instability strip. As they evolve they increase their temperature, and hence fundamental pulsating RR0 Lyrae would develop into first-overtone RR1 variables. In contrast, the horizontalbranch stars in the more metal-poor OoII clusters approach the instability strip from a different direction that those in the intermediate metallicity clusters. They begin their lives at higher temperatures and evolve to lower temperatures. The existence of an hysteresis zone is suggested by van Albada \& Baker (1971), so that depending on the direction of evolution, mode switching is delayed and occurs at different temperatures. Using convective pulsating models as well as observations of horizontal-branch stars in two different Oosterhoff GCs, Bono, Caputo \& Marconi (1995) were able to show that the transition between RR0 and RR1 RR Lyrae supports the hysteresis mechanism.

The van Albada \& Baker (1971) explanation thus suggests (1) OoI clusters are evolving red to blue and OoII clusters the other way, (2) that OoI clusters have a smaller fraction of RR1 variables than OoII clusters, and (3) that metallicity is the dominant characteristic that determines where on the horizontal-branch core helium burning begins. However, this explanation does not explain the absence of a dichotomy in other galaxies unless the range in metallicity in each galaxy is very small (Lee \& Carney 1999).

Ultimately, the issue of the second parameter problem in the horizontal-branch morphology could potentially account for the Oosterhoff dichotomy. Bluer and more extended HBs exist in the more metal-poor clusters while metal-rich ones have more of a red clump (Lee, Demarque, \& Zinn 1990). Yet as this is not always the case, there must be another factor, the second parameter, which affects HB morphology. Two possibilities of this second parameter could be helium enrichment through a variety of mechanisms (Sweigart \& Catelan 1998) and age. Now with the discovery of metal-rich bulge GCs that do not follow the period-metallicity trends of the other two groups (Pritzl et al. 2001), the second parameter issue is of considerable importance to understand these "Oosterhoff III" GCs.

The Oosterhoff dichotomy was first seen in GCs, but there has been quite a bit of interest to see how field RR Lyrae stars pertain to the Oosterhoff dichotomy. Recently using RR0 Lyrae stars in the LONEOS-I, SDSS-II Supernova Survey and All Sky Automated Survey (ASAS) two distinct OoI and OoII components were found by examining the RR Lyrae period shift (Miceli et al. 2008; de Lee 2008; Szczygiel et al. 2009). The LONEOS-1 survey covers $1430 \mathrm{deg}^{2}$ to a distance of 30 $\mathrm{kpc}$ and traces the ecliptic. The SDSS-II Supernova Survey covers a $2.5^{\circ}$ equatorial stripe ranging from -60 to +60 degrees in RA, and covers a distance of 3 to $95 \mathrm{kpc}$ from the sun. ASAS surveys the southern sky up to $\delta=+28$ and reaches to distance of $\sim 5 \mathrm{kpc}$. 
In contrast, the QUEST-I RR Lyrae (Vivas et al. 2004) and the NSVS RR Lyae (Kinemuchi et al. 2006) did not show a clear OoII component. The NSVS survey was recently shown to have incorrect $V$-amplitudes, which concealed the presence of two Oosterhoff components in their sample (Szczygiel et al. 2009). The NSVS survey spans a region of $5 \mathrm{kpc}$ from the Sun and primarily covers the entire northern sky. The QUEST RR Lyrae survey covers $380 \mathrm{deg}^{2}$ to a distance of 55 $\mathrm{kpc}$, and covers equatorial regions of the sky. We investigate here if the Oosterhoff dichotomy is present in the field Galactic bulge RR0 Lyrae stars. This region is distinct from the LONEOS-I, QUEST, ASAS, SDSS-II and NSVS surveys. Further, this region has metal-rich RR Lyrae stars, which, except for the recently discovered "OoIII" clusters mentioned above, are hardly seen in the Milky Way Globular Clusters.

\section{Data}

We consider the RR0 Lyrae stars from the MACHO bulge fields with "normal" light curves (Kunder et al. 2008). A "normal" light curve was first described by Jurcsik \& Kovács (1996) who used interrelations among the lower order Fourier parameters to derive a set of equations for calculating Fourier amplitudes and phase differences. A star's light curve is considered "normal" if the calculated values for all of these parameters are in good agreement with the observed values

and hence has a low deviation parameter. Since then, Kovács \& Kanbur (1998) have updated the deviation parameter analysis, and their values for deviation parameters are used in this analysis. The stars from Kunder \& Chabover (2008) have published photometric [Fe/H] metallicities determined from their Fourier decomposition parameters as well as reddening estimates as determined from their $(\mathrm{V}-\mathrm{R})$ color at minimum $V$-band light.

Only "normal" RR Lyrae variables are used because the Period-Amplitude $\left(P A_{V}\right)$ plane, which is often used to distinguish between Oosterhoff type I and Oosterhoff type II globular clusters, becomes contaminated by Blazhko variables. Blazhko variables are RR Lyrae stars with non-repeating light curves, and hence their amplitudes vary over timescales longer than the basic pulsation period. If not identified, they introduce scatter into the PAV diagram. Using those stars with "normal" light curves significantly reduces a major contributor of contamination in the $\mathrm{PA}_{\mathrm{V}}$ diagram.

To further asses the quality of the light curves, all the "normal" light curves were visually inspected and the stars with the most high quality light curves were selected. Stars that exhibit the Blazhko effect have especially notable scatter at the height of maximum of their light curves, and hence, we particularly checked that the scatter around maximum light was minimal, or at least consistent with the scatter of the light curve. This resulted in a sample of 1323 high quality light curves. Examples of three typical high quality and three typical standard light curves are shown in the left and right panels of Figure 1,

We now discuss the reliability of a MACHO RR Lyrae stars' period and $V$-amplitude measurement; hence the reliability of a MACHO RR Lyrae's location on a PAV diagram can be assessed. 
An RR0 Lyrae stars' period is perhaps the most well determined parameter, especially within the 8 year MACHO dataset. From internal MACHO comparisons, the error in period is $\sigma_{\mathrm{P}}=0.000054^{\mathrm{d}}$ (Minniti 1996).

The observed $V$-amplitude of the RR Lyrae stars used in this analysis is found by performing an eighth order Fourier decomposition to their light curves. This in contrast to the amplitudes listed

by Kunder et al. (2008) which are derived from a fourth order Fourier decomposition. However, an eighth order Fourier fit better represents the minimum and maximum extremes of the RR Lyrae light curve, which is used to determine amplitude. The fourth order amplitudes begin to deviate from eighth order amplitudes at $A_{V}<1.0 \mathrm{mag}$ and are on average $\sim 0.02$ to 0.03 mag larger than the 8th order amplitudes.

A check on the accuracy of the MACHO RR0 Lyrae amplitudes is performed by using the LMC RR0 Lyrae stars in the OGLE-II and MACHO databases. Both surveys list RR0 Lyrae $V$-amplitudes.

From a sample of 467 OGLE-II and MACHO LMC RR0 Lyrae stars, the mean difference between the OGLE $V$-amplitudes and the MACHO $V$-amplitudes is $0.05 \pm 0.13 \mathrm{mag}$, where 0.13 refers to the dispersion about the mean. The distribution is roughly Gaussian. As the dispersion is quite a bit larger than the mean, the MACHO and OGLE $V$-amplitudes agree reasonably well with each other. There is likely error in the OGLE $V$-amplitudes as well as the MACHO $V$-amplitudes, so the random error in MACHO $V$-amplitude is $0.10 \mathrm{mag}$, and the zero-point in MACHO $V$ amplitude is up to $0.05 \mathrm{mag}$. To diminish the random error, much of the analysis is presented in the form of averages.

The MACHO bulge RR Lyrae light curves are of better quality than the MACHO LMC RR Lyrae light curves. The LMC RR Lyrae light curves exhibit more scatter than the bulge RR Lyrae stars. The LMC RR Lyrae stars are considerably fainter, with an average brightness of $V=19.4$ and a mean error of a single point on the light curve is $\pm 0.07 \mathrm{mag}$ (Alcock et al. 1996). This is in contrast to the bulge RR Lyrae stars which have magnitudes that peak at $V=15$ mag (Alcock et al. 1997) and the mean error of a single point on the light curve is \pm 0.02 mag. As a result, the discussion in the previous paragraph likely overestimates the $V$-amplitude error.

\section{Period-Amplitude Plane}

Sandage (1982) showed that a better parameter for the Oosterhoff classification is not the average of all the RR0 Lyrae periods but the measurement of, in the period-blue amplitude relationship, the difference between the average periods of an M3 RR Lyrae and the observed period at a fixed blue amplitude $A_{B}$. This is given by

$$
\Delta \log P=0.129 A_{B}+0.088-\log P(\text { observed }) .
$$

Since then, this period shift has also been defined as a function of $V$-amplitude. The least-squares 
fit to RR0 stars in the OoI prototype GC M3 is

$$
\Delta \log P=-0.14 A_{V}-0.12-\log P(\text { observed })
$$

and the least-squares fit to RR0 stars in the OoII GC M9 is

$$
\Delta \log P=-0.20 A_{V}+0.026-\log P(\text { observed })
$$

(Clement \& Shelton 1999).

Figure 2 shows the Period-Amplitude $\left(\mathrm{PA}_{\mathrm{V}}\right)$ diagram of the 1323 high quality MACHO bulge RR0 Lyrae variables in the bottom panel and the 1028 standard MACHO bulge RR Lyrae in the top panel. Overplotted are the lines that Clement \& Shelton (1999) derived for Oosterhoff II and Oosterhoff I RR0 stars. In both panels it is clear that the majority of MACHO bulge field RR Lyrae do not fall along either of these lines, nor is there a "gap" between these lines. The amplitudes would have to be shifted by $\sim 0.2 \mathrm{mag}$ for the main locus of stars to follow the OoI line. From the above amplitude analysis, it is unlikely for the MACHO $V$-band amplitudes to be this discrepant. There is considerable scatter in this plot, although the main locus of the high quality RR Lyrae shows less scatter. The amplitude of light variation for Blazhko stars varies over timescales longer than the basic pulsation period and introduce scatter into the $\mathrm{PA}_{\mathrm{V}}$ diagram if not identified. Since only RR0 Lyrae stars with "normal" light curves are used here, there should not be many Blazhko variables introducing scatter to this plot. Further, only those with no clear indication of the Blazhko effect were selected to be part of the high quality MACHO RR Lyrae. This strengths the result that their is no gap in the Bulge field RR0 Lyrae stars.

It is notable that the $\mathrm{PA}_{\mathrm{V}}$ relation for the stars with shorter periods (i.e. OoI characteristics), appear to follow a quadratic relation more than a linear relation. This was also pointed out by Cacciari et al. (2005) with their study of the OoI characteristic M3 RR Lyrae stars. Their quadratic $\mathrm{PA}_{\mathrm{V}}$ relation is not used here because it does not give a solution for the period of an RR Lyrae star with an $A_{V}$ greater than $1.31 \mathrm{mag}$, and there are RR0 Lyrae stars with a $V$-band amplitude greater than $1.31 \mathrm{mag}$ in this sample. As seen in Figure 2, the main locus of MACHO RR Lyrae stars does not fall on this line either. There are $\sim 20$ variables with $V$-band amplitudes greater than 1.31 mag in this sample. This again illustrates the differences between the properties of the RR Lyrae variables in GCs compared to those located in the Galactic bulge.

\section{4. $\quad \Delta \log \mathrm{P}$ As An Oosterhoff Indicator}

The period shift defined by Equation 3, $\Delta \log \mathrm{P}$, is usually made between the RR0 Lyrae stars in different globular clusters (Sandage 1981; Sandage et al. 1981; Carney et al. 1992; Sandage 1993 ). This quantity can be used as an Oosterhoff indicator. For example, when finding the period shift with respect to the OoI line, a period shift of $\Delta \log \mathrm{P}=0$ would indicate an Oosterhoff type I star, and a period shift of $\Delta \log \mathrm{P} \sim-0.07$ would indicate an Oosterhoff type II star. Those values in-between show the degree of deviation of an RR Lyrae star from an Oosterhoff class. 
Suntzeff, Kinman \& Kraft (1991) plot the period shift, $\Delta \log$, with respect to the OoI line against metallicity using a sample of 171 RR0 field stars with spectroscopically determined metallicities. These stars are in the galactocentric distance range $4-30 \mathrm{kpc}$ and are at located from $(l, b)$ $=\left(1\right.$ to $182^{\circ},-18$ to $\left.+89^{\circ}\right)$. They found a gap at $\Delta \log \mathrm{P} \sim-0.03$, and hence a clear separation between field stars of Oosterhoff type I and II. Figure 3 shows the period shifts of the high quality MACHO RR0 Lyrae stars as a function of their photometric metallicities. There is no evidence of a gap.

It is striking how many RR Lyrae stars in the bulge sample extend to $\Delta \log \mathrm{P}>0.05$, a property not common in RR0 Lyrae stars in globular clusters. These stars are on average more metal-rich than the rest of the sample, and such metal-rich globular clusters are scarce in the Milky Way. The RR0 Lyrae stars in the metal-rich globular clusters, NGC 6441 and NGC 6388 (with [Fe/H] -0.6 ) have $\Delta \log \mathrm{P}$ values that fall in the OoII region, with $\Delta \log \mathrm{P}$ values of $\sim-0.09$ days (despite their high metallicity). The $\Delta \log \mathrm{P}$ values for these variables are plotted in Figure 3. It is clear that the metal-rich RR Lyrae field stars in the bulge have quite different $\Delta \log \mathrm{P}$ values than that of the metal-rich globular cluster RR Lyrae stars. NGC 6388 and NGC 6441 have been found to be more consistent with Oosterhoff type II clusters than Oosterhoff type I clusters. However, for the metal-rich stars in the Galactic bulge, this is not the case. This strongly suggests that the metal-rich RR Lyrae stars in the Galactic bulge may have a different origin than that in the metal-rich globular clusters.

In Figure 4, the distribution of the period shift, $\Delta \log \mathrm{P}$, at fixed $V$-band amplitude from the OoI line is shown. Only the RR Lyrae with high quality light curves are used here. However, this histogram looks almost identical if all the MACHO bulge stars are used. Also shown are the $\Delta \log \mathrm{P}$ value from the OoI clusters M3 and M5, as well as the $\Delta \log \mathrm{P}$ values from the OoII cluster M2 and M9. The bulge RR0 Lyrae stars have $\Delta \log P$ distributions that clearly do not coincide with either OoI or OoII distributions. This is not surprising, as this was evident in the PA-plane as well. The main locus of the distributions are shifted by $\Delta \log \mathrm{P} \sim 0.02$ days relative to the OoI and OoII designations.

Our survey of bulge RR Lyrae stars have found that the stars do not fit into the classic OoI and OoII designations (with a period shift of 0.025 days), and indeed there is no evidence for an Oosterhoff dichotomy in our work. In contrast, almost all of the recent surveys of field RR Lyrae stars, i.e., LONEOS (Miceli et al. 2008), SDSS-II Supernova Survey (de Lee 2008), ASAS (Szczygiel et al. 2009), have found that the majority of their RR Lyrae $\Delta \log P$ values correspond well to the OoI $\Delta \log \mathrm{P}$ peak. Moreover, these surveys have also identified a displaced secondary peak corresponding to an OoII component. The QUEST-I sample (Vivas et al. 2004) and the NSVS sample (Kinemuchi et al. 2006), however, find no gap in their PA-diagram distributions. Szczygiel et al. (2009) shows that the NSVS $V$-amplitudes are incorrect, which had the effect of masking the two Oosterhoff components in their sample. THE QUEST RR Lyrae catalog, which did not find a Oosterhoff dichotomy in their sample, probes deeper than the LONEOS survey (by $\sim 25 \mathrm{kpc}$ ), and hence would give a more complete picture of the Galactic halo. However, OoII RR 
Lyrae stars in general are located closer to the Galactic Plane (Lee \& Carney 1999; de Lee 2008), and hence the depth of the QUEST survey may have the effect of limiting the number of OoII RR Lyrae with respect to OoI RR Lyrae. None of these samples probe the Galactic bulge, and are located at quite different locations on the sky as the MACHO bulge RR0 Lyrae sample.

\section{1. $\quad \Delta \log \mathrm{P}$ and $V$-amplitude for a given period}

Clement \& Shelton (1999) argue that for a given period, the $V$-amplitude (a fairly good temperature indicator) for "normal" RR0 Lyrae stars does not depend on $[\mathrm{Fe} / \mathrm{H}]$, but on Oosterhoff type. They use seven globular clusters to come to this conclusion and apply the same compatibility test as Kunder \& Chabover (2008) to establish which cluster RR0 Lyrae stars had peculiar light curves. Clement \& Shelton (1999) then present evidence that the Oosterhoff type of an RR Lyrae star is a function of its evolutionary state. If these arguments hold, Clement \& Shelton (1999) discuss the implications on the determination of the ages of globular clusters from RR Lyrae stars. We use the Galactic bulge to investigate this notion.

The RR Lyrae variables are binned in 0.01 day bins and the average $[\mathrm{Fe} / \mathrm{H}], V$-amplitude and $\Delta \log \mathrm{P}$ of the RR Lyrae in each period bin is found. Figure 5 shows the average $A_{V}$ in each period bin as a function of both $[\mathrm{Fe} / \mathrm{H}]$ and $\Delta \log \mathrm{P}$. For clarity, only the RR0 Lyrae period bins with periods less than 0.62 day are shown. It is clear from the bottom panel of Figure 5 that the average $V$-amplitude in each period bin does not depend on metallicity. In contrast, their is a slope in the $A_{V}$ and $\Delta \log \mathrm{P}$ plane for the OoI-like RR0 Lyrae stars. This slope is $0.11 \pm 0.02$ days $/ \mathrm{mag}$. With the exception for the most metal-rich stars, the stars with shorter periods $(\mathrm{P}<0.5 \mathrm{~d})$ show this result as well. Many studies have used the position of an RR Lyrae star in the period- $V$-amplitude diagram to estimate its metallicity (Alcock et al. 1996, 2000; Kinemuchi et al. 2006; Sandage 2004; Brown et al. 2004). Here strong evidence is presented that cautions against using the period and $V$-amplitude of an RR Lyrae star to estimate its metallicity. A similar analysis cannot be carried out with the OoII-like RR0 Lyrae stars, because they exhibit a wide range of metallicities with no clear function on $V$-amplitude for a given period.

\section{5. $\quad \Delta \log \mathrm{P}$ and Luminosity Differences}

Lee \& Carney (1999) performed an investigation on the luminosities of RR Lyrae variables with similar metallicities but different Oosterhoff classes. They found that RR Lyrae variables in the Oosterhoff II cluster M2 are intrinsically brighter than those in Oosterhoff group I cluster M3, although these clusters have similar $[\mathrm{Fe} / \mathrm{H}]$. Thus at least in this cluster pair, there is a discontinuity in the $\mathrm{M}_{\mathrm{V}}^{\mathrm{RR}}-[\mathrm{Fe} / \mathrm{H}]$ relation. Here a similar analysis is carried out using first the bulge field RR0 Lyrae stars and second the local field RR0 Lyrae stars. 


\subsection{Bulge Field RR0 Lyrae Star Luminosities}

Figure 6 shows the $\Delta \log \mathrm{P}$ distributions for the bulge field RR0 Lyrae stars with different metallicity ranges. The high quality RR Lyrae stars are represented by the dotted lines, and have very similar $\Delta \log \mathrm{P}$ distributions to the complete sample. Unlike for the most metal-rich RR0 Lyrae stars in the bulge field RR0 Lyrae sample, the most metal-poor RR0 Lyrae samples have stars that occupy both the OoI and OoII regions as indicated by their $\Delta \log \mathrm{P}$.

The reddening-free apparent magnitude of the RR0 Lyrae variables with high quality light curves and photometric metallicities of $-1.7<[\mathrm{Fe} / \mathrm{H}]<-1.5$ is shown in Figure 7 as a function of $\Delta \log \mathrm{P}$. The Wesenheit reddening free magnitude, $W_{0}$, is used to avoid uncertain reddening corrections and is defined as $W_{0}=V-4.3(V-R)$, where the factor 4.3 is the selective extinction coefficient $R_{V, V R}$ derived by Kunder et al. (2008). There are 107 stars with metallicities between $[\mathrm{Fe} / \mathrm{H}]=-1.5$ dex and $[\mathrm{Fe} / \mathrm{H}]=-1.7$ dex, and their distribution in the $\Delta \log \mathrm{P}$ plane is shown in the bottom panel of Figure 6. It is striking that this rather metal-poor sample has a $\Delta \log \mathrm{P}$ distribution with an OoI-like tail. Dividing the RR0 Lyrae at $-0.03 \Delta \log \mathrm{P}$ days, the average $W_{0}$ for the stars with $\Delta \log \mathrm{P}<-0.03 \mathrm{~d}$, or the OoII-like stars, is $W_{0, I I}=14.33 \pm 0.07 \mathrm{mag}$. The average $W_{0}$ for the stars with $0.06 ; \Delta \log \mathrm{P}>-0.03$, or the OoI-like stars, is $W_{0, I}=14.58 \pm 0.07$ mag. No bias in location is found for this particular sample of stars $-\mathrm{W}_{0}$ and $\Delta \log \mathrm{P}$ is evenly distributed as a function of Galactic $l$ or $b$. This is consistent with Kunder \& Chaboyer (2008), in which it is shown that for the entire sample of bulge stars, there is no strong evidence of $\mathrm{W}_{0}$ as a function of Galacitic $l$ or $b$. As there is no trend in $[\mathrm{Fe} / \mathrm{H}]$ as a function of $\Delta \log \mathrm{P}$, this apparent magnitude difference shows that there is a discontinuity in the absolute magnitude- $[\mathrm{Fe} / \mathrm{H}]$ relation for this particular sample of stars.

This same analysis is carried out with the 320 bulge field RR0 Lyrae stars that have metallicities in the $-1.5<[\mathrm{Fe} / \mathrm{H}]<-1.3$ dex range. Again, only the stars with high quality light curves are used. Figure 8 shows how the reddening-free apparent magnitude decreases as the RR0 Lyrae stars display more OoI-like $\Delta \log \mathrm{P}$ values. For this metallicity distribution, there are a handful of stars at $\Delta \log \mathrm{P}>0.06$ days. These stars were shown to have $V$-band amplitudes that were more similar to the $A_{V}$ found in the OoII-like stars rather than the $A_{V}$ found in the OoI-like stars. In Figure 8 it is evident that their $\left\langle\mathrm{W}_{0}>\right.$ become more luminous than those OoI-like stars at $\Delta \log \mathrm{P}<0.06$ days. The average $W_{0}$ for those stars with $\Delta \log \mathrm{P}<-0.03$ days is $W_{0, I I}=14.44 \pm 0.07 \mathrm{mag}$. The average $W_{0}$ for the stars with $\Delta \log \mathrm{P}>-0.03$ is $W_{0, I}=14.56 \pm 0.03$ mag. Disregarding the stars with $\Delta \log \mathrm{P}>0.06$ days, the average $W_{0}$ is $W_{0, I}=14.55 \pm 0.03$ mag.

The stars with high quality light curves in the $-1.3<[\mathrm{Fe} / \mathrm{H}]<-1.1$ dex metallicity range show similar effects. There are not enough stars in the more metal-rich or more metal-poor metallicity ranges to perform such an analysis. Table 1 summarizes these results. When using all the RR Lyrae variables and not just those with high quality light curves, the same effect is seen. Further, when all the light curves are used, one more metal-rich and one more metal-poor range can be investigated. Both of these metallicity ranges show that the OoII-type Bulge stars have brighter reddening-free 
magnitudes than OoI-type RR Lyrae. This indicates that unless the RR0 Lyrae absolute magnitude relation includes a period-amplitude term, the relation can lead to uncertainties of up to $0.2 \mathrm{mag}$ in absolute magnitude. Furthermore, a quadratic $\mathrm{M}_{\mathrm{V}}^{\mathrm{RR}}-[\mathrm{Fe} / \mathrm{H}]$ relation would not correct for this.

\subsection{Local Field RR0 Lyrae Star Luminosities}

The previous section suggests that $[\mathrm{Fe} / \mathrm{H}]$ alone cannot account for absolute magnitudes of RR Lyrae variables, and that an RR Lyrae variables position on the $P A_{V}$ diagram may influence the absolute magnitude. How robust this result is for local RR Lyrae stars is also of interest. Recently Kovács (2003) used the observed $V$-band, $(V-K)$ colors and radial velocity curves of local RR Lyrae stars to compute their luminosity, effective temperature, and radius variations. The results of this Baade-Wesselink (BW) analysis is presented in his Table 1. $V$-amplitudes from the literature are obtained for these same stars, (Sandage 2004, Jones, Carney \& Latham 1988, Kunder et al.(2008), Lub 1977) and their $\Delta \log \mathrm{P}$ was computed. $[\mathrm{Fe} / \mathrm{H}]$ values for these stars come from Layden et al. (1996).

Bono et al. (2003) compiled a list of 36 RR0 Lyrae stars (field and GGC) for which accurate $V$ and $K$ light curves, reddening and metallicity estimates were known. From these parameters and their updated pulsation models, the $M_{K}$ absolute magnitude was derived and in turn, the $M_{V}$ absolute magnitude. $\Delta \log \mathrm{P}$ values for 15 stars listed in the Bono et al. (2003) table that are not present in the Kovács (2003) sample are obtained.

Figure 9 shows the absolute magnitude of 36 resulting RR Lyrae stars as a function of $[\mathrm{Fe} / \mathrm{H}]$ and $\Delta \log \mathrm{P}$. Kovács $(2003)$ and Bono et al. (2003) do not list an error in $\mathrm{M}_{\mathrm{V}}^{\mathrm{RR}}$, but the standard methodology of the BW analysis typically results in errors in absolute magnitude of 0.2 mag (Liu \& Janes 1990). It is also evident with the local field RR Lyrae stars with $\Delta \log \mathrm{P}<-0.03$ days, (i.e. the OoII-like stars) have on average brighter absolute magnitudes.

There is a cluster of seven stars with $[\mathrm{Fe} / \mathrm{H}]$ of $\sim-1.75$ dex, and these stars have a range in absolute magnitudes of 0.6 mag. This is indication that a second parameter may influence the absolute magnitude of an RR Lyrae. It is especially striking that the five RR Lyrae stars with the brightest absolute magnitudes, represented in Figure 9 by filled triangles, introduce large scatter in the $[\mathrm{Fe} / \mathrm{H}]$ vs $\mathrm{M}_{\mathrm{V}}^{\mathrm{RR}}$ plot, but follow the $\Delta \log \mathrm{P}$ versus $\mathrm{M}_{\mathrm{V}}^{\mathrm{RR}}$ trend reasonably well. These stars especially highlight that an RR Lyrae variable's metallicity may not always indicate its absolute magnitude. When determining absolute magnitude, the stars' $\Delta \log \mathrm{P}$ must be taken into account.

A least-squares fit to $[\mathrm{Fe} / \mathrm{H}]$ versus $\mathrm{M}_{\mathrm{V}}^{\mathrm{RR}}$ results in a dispersion about the fit of 0.19 , and the least-squares fit of $\Delta \log \mathrm{P}$ versus $\mathrm{M}_{\mathrm{V}}^{\mathrm{RR}}$ results in a dispersion about the fit of 0.13 . A simultaneous fit of $[\mathrm{Fe} / \mathrm{H}]$ and $\Delta \log \mathrm{P}$ versus absolute magnitude also results in a dispersion about the fit of 0.15. The BW stars have $\Delta \log \mathrm{P}$ values that are tightly correlated with $[\mathrm{Fe} / \mathrm{H}]$ and hence it is difficult to disentangle the effect of absolute magnitude versus $\Delta \log \mathrm{P}$ and $[\mathrm{Fe} / \mathrm{H}]$. 
Although most RR Lyrae variables are found in relatively metal-poor globular clusters, two metal-rich GCs, NGC 6388 and NGC 6441, have been found to host RR Lyrae variables. These clusters have very unusual HB morphologies for their metallicities (Rich et al. 1997). Although NGC 6388 and NGC 6441 have metallicities of $[\mathrm{Fe} / \mathrm{H}] \sim-0.6$ dex, which are indicative of OoI GCs, their $\Delta \log \mathrm{P}$ values fall inline with the OoII GCs, with $\Delta \log \mathrm{P} \sim-0.09$ days. Traditionally, their $[\mathrm{Fe} / \mathrm{H}]$ would indicate that these stars are intrinsically fainter than most other RR Lyrae variables. However, models introduced by Sweigart \& Catelan (1998) require that the HBs of these clusters be unusually bright for the metal abundances of the clusters. Figure 8 shows that these stars should be intrinsically brighter than most RR Lyrae variables, because of their values of $\Delta \log \mathrm{P}$.

\section{Conclusion}

Field bulge RR0 Lyrae stars are used to search for evidence of the Oosterhoff dichotomy within the field population in the Milky Way halo. The period- $V$-amplitude plane for the bulge field RR0 Lyrae stars does not have a locus that matches either the OoI or OoII period- $V$-amplitude plane of the MW globular clusters. The bulge field RR0 Lyrae stars are in general more metal-rich than even the OoI-type GCs. Consequently, their main locus is shifted in the same manner as the OoI-type GCs is shifted compared to the more metal-poor OoII-type GCs.

The metal-rich bulge RR Lyrae variables lie on a different period- $V$-amplitude plane than the metal-rich GC RR0 Lyrae stars in NGC 6388 and 6411. The metal-rich GC RR0 Lyrae stars have $P A_{V}$ properties more similar to the OoII-type GC RR0 Lyrae stars, whereas this is reversed for the metal-rich bulge RR Lyrae variables. It is unlikely that the metal-rich RR Lyrae stars in the Galactic bulge and the metal-rich RR Lyrae stars in NGC 6388 and NGC 6411 come from a similar origin.

Although the period-amplitude relations for OoI and OoII-type GCs use linear forms to determine the period shift, $\Delta \log \mathrm{P}$, of a GC, the period- $V$-amplitude plane of the thousands of Galactic bulge RR0 Lyrae stars show that a quadratic period- $V$-amplitude relation would be a better approximation. Cacciari et al. (2005) found this with RR0 Lyrae variables in M3. However, their relation does not give a solution for the period of an RR Lyrae star with a $V$-amplitude greater than 1.31 mag. There are bulge field RR0 Lyrae stars with a $V$-band amplitude greater than 1.31 mag.

The reddening-free apparent magnitude differences between the OoI and OoII-type bulge field RR Lyrae stars are discussed. As was found in RR0 Lyrae stars in GCs (Lee \& Carney 1999), RR0 Lyrae variables with OoII-type properties are about 0.2 mag brighter than those with OoItype properties. The differences in the evolutionary stages of the OoI and OoII-type variables are

probably the cause for this luminosity difference. A quadratic dependence of $[\mathrm{Fe} / \mathrm{H}]-\mathrm{M}_{\mathrm{V}}^{\mathrm{RR}}$ would not correct this effect. Using local field RR Lyrae stars with absolute magnitude values determined 
from a Baade-Wesselink analysis, it is shown that $\Delta \log \mathrm{P}$ is a better indicator of an RR0 Lyrae stars' absolute magnitude than $[\mathrm{Fe} / \mathrm{H}]$. 


\section{REFERENCES}

Alcock, C., Allsman, R.A., Axelrod, T.S., Bennett, D.P. Cook, K.H., Freeman, K.C., Griest, K., Marshall, S.L, Peterson, B.A., Pratt, M.R., Quinn, P.J., Rodgers, A.W., Stubbs, C.W., Sutherland, S., \& Welch, D.L. 1996, AJ, 111, 3

Alcock, C. et al. 1997, ApJ, 486, 697

Alcock, C., et al. 2000, AJ, 119, 2194

Brown, T. M., Ferguson, H., Smith, E., Kimble, R. A., Sweigart, A. V., Renzini, A., \& Rich, R. M. 2004, AJ, 127, 2738

Bono, G., Caputo, F., Castellani, V., Marconi, M., Storm, J., \& Degl'Innocenti, S. 2003, MNRAS, 344,1097

Bono, G., Caputo, F. \& Marconi, M. 1995, AJ, 110, 2365

Bono, G., Caputo, F., di Criscienzo, M. 2007, A\&A, 476, 779

Cacciari, C., Corwin, T. M., \& Carney, B. W. 2005, AJ, 129, 267

Carney, B. W., Storm, J., \& Jones, R. V. 1992, ApJ, 386, 663

Carretta, R.G., Gratton, G., Clementini, F., Fusi Pecci, ApJ, 533, 215

Catelan, M. 2005, in Resolved Stellar Populations, ASP Conf. Ser., ed. D. Valls-Gabaud \& M. Chávez (San Francisco: ASP), in press

Catelan, M. 2007, in Globular Clusters: Guides to Galaxies, ed. D. Geisler \& T. Richtler (ESO; Berlin: Springer)

Clement, C.M. \& Shelton, I. 1999, ApJ, 515, 88

Clementini, G. Gratton, R. Bragaglia, A., Ripepi, V., Fiorenzano, A.F.M., Held, E.V. \& Carretta, E. 2005, ApJ, 630L, 145

de Lee, N. 2008, Ph.D. thesis, 10, Michigan State University

Eggen, O. J., Lynden-Bell, D., \& Sandage, A. R. 1962, ApJ, 136, 748

Jones, R.V., Carney, B.W. \& Latham, D.W. 1998, ApJ, 332, 206

Jurcsik, J., \& Kovács, G. 1996, A\&A, 312, 111

Kinemuchi, K., Smith, H.A., Woźniak, P.R., McKay, T.A., \& The ROTSE Collaboration 2006, AJ, 132, 1202

Kovács, G., \& Kanbur, S. M. 1998, MNRAS, 295, 834 
Kovács, G. 2003, MNRAS, 342, 58

Kunder, A.M., Popowski, P., Cook, K.H., \& Chaboyer, B. 2008, AJ, 135, 631

Kunder, A.M. \& Chaboyer, B. 2008, AJ, 135, 631

Layden, A.C., Hanson, R.B., Hawley, S.L., Klemola, A.R. \& Hanley, C.J. 1996, AJ, 112, 5

Lee, J. \& Carney, B.W. 1999, AJ, 118, 1373

Lee, Y.-W., Demarque, P., \& Zinn, R. 1990, ApJ, 350, 155

Liu, T. \& Janes, K.A. 1990, ApJ, 360, 561

Lub, J. 1977, A\&AS, 29, 345

Minniti, D. 1996, ApJ. 459. 175

Miceli, A., Rest, A., Stubbs, C.W., Hawley, S.L., Cook, K.H., Magnier, E.A., Krisciunas, K., Bowell, E., \& Koehn, B. 2008, ApJ, 678, 865

Oosterhoff, P. Th. 1939, Observatory 62, 104

Pritzl, B. J., Smith, H. A., Catelan, M., \& Sweigart, A. V. 2001, AJ, 122, 2600

Pritzl, B. J., Smith, H. A., Stetson, P.B., Catelan, M., Sweigart, A. V., Layden, A.C. \& Rich, R.M. 2003, AJ, 126, 1381

Pritzl, B. J., Armandroff, T. E., Jacoby, G. H., \& Da Costa, G. S. 2004, AJ, 127, 318

Rich, R. M., et al. 1997, ApJ, 484, L25

Sandage A. 1958, ApJ, 127, 515

Sandage, A. 1981, ApJ, 248, 161

Sandage, A. 1982, ApJ, 252, 574

Sandage, A. 1993, AJ, 106, 687

Sandage, A. 2004, AJ, 128, 858

Sandage, A. 2006, AJ, 131, 1750

Sandage, A., Katem, B., \& Sandage, M. 1981, ApJS, 46, 41

Sandage, A., \& Tammann, G. A. 2006, ARA\&A, 44, 93

Searle, L., \& Zinn, R. 1978, ApJ, 225, 357 
Siegel, M. H., \& Majewski, S. R. 2000, AJ, 120, 284

Suntzeff, N., Kinman, T, \& Kraft, R. 1991, ApJ, 367, 528

Sweigart, A. V., \& Catelan, M. 1998, ApJ, 501, L63

Szczygiel, D.M. (in prep.)

van Albada, T.S., \& Baker, N., 1973, ApJ, 185, 477

Vivas, A. K., et al. 2004, AJ, 127, 1158

Walker, A.R.: in IAU Colloq. 176, The Impact of Large-Scale Surveys on Pulsating Star Research, ed. L. Szabados \& D. Kurtz. (San Francisco: ASP), 165 (2000) 
Table 1: Apparent Magnitude Differences Between Oosterhoff I-like and Oosterhoff II-like bulge RR0 Lyrae Field Stars

\begin{tabular}{lcccccccc}
\hline Metallicity Bin & $\left\langle\mathrm{W}_{0, \mathrm{II}}\right\rangle$ & $\left\langle\mathrm{W}_{0, \mathrm{I}}\right\rangle$ & $\left\langle[\mathrm{Fe} / \mathrm{H}]_{\mathrm{II}}\right\rangle$ & $\left\langle[\mathrm{Fe} / \mathrm{H}]_{\mathrm{I}}\right\rangle$ & $\left\langle\mathrm{P}_{\mathrm{II}}\right\rangle$ & $\left\langle\mathrm{P}_{\mathrm{I}}\right\rangle$ & $\left\langle\mathrm{A}_{\mathrm{V}_{\text {II }}}\right\rangle$ & $\left\langle\mathrm{A}_{\mathrm{V}_{\mathrm{I}}}\right\rangle$ \\
\hline$-1.7<[\mathrm{Fe} / \mathrm{H}]<-1.5$ & $14.33 \pm 0.07$ & $14.58 \pm 0.07$ & $-1.60 \pm 0.01$ & $-1.58 \pm 0.01$ & $0.62 \pm 0.01$ & $0.53 \pm 0.01$ & $1.02 \pm 0.02$ & $1.04 \pm 0.03$ \\
$-1.5<[\mathrm{Fe} / \mathrm{H}]<-1.3$ & $14.44 \pm 0.07$ & $14.55 \pm 0.03$ & $-1.40 \pm 0.01$ & $-1.37 \pm 0.01$ & $0.62 \pm 0.01$ & $0.52 \pm 0.00$ & $0.95 \pm 0.03$ & $1.04 \pm 0.01$ \\
$-1.3<[\mathrm{Fe} / \mathrm{H}]<-1.1$ & $14.33 \pm 0.06$ & $14.53 \pm 0.02$ & $-1.21 \pm 0.01$ & $-1.21 \pm 0.00$ & $0.63 \pm 0.01$ & $0.53 \pm 0.00$ & $0.84 \pm 0.03$ & $0.98 \pm 0.01$ \\
\hline
\end{tabular}



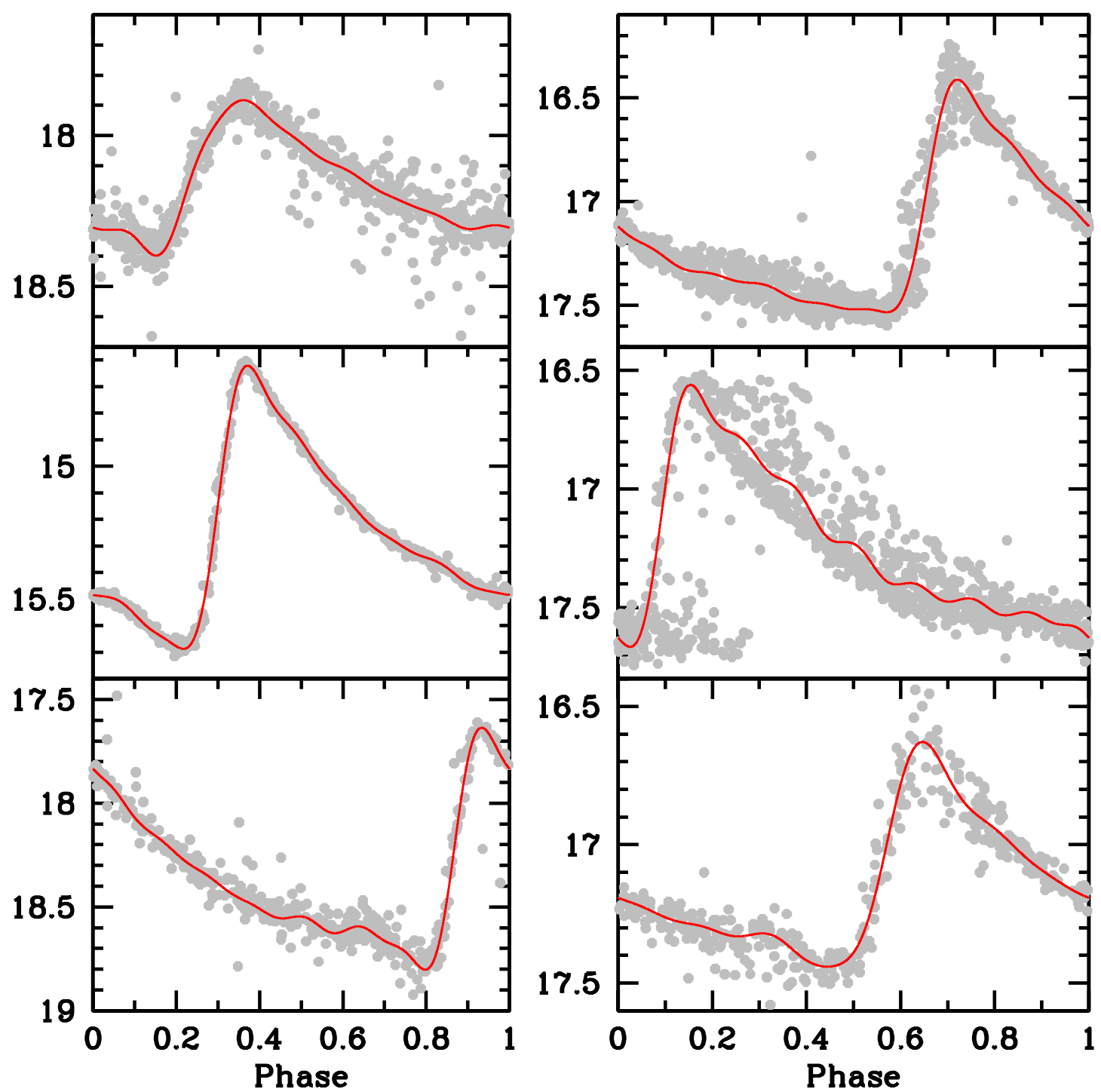

Fig. 1. - Left: Three of the 1323 high quality MACHO bulge light curves. From top to bottom these stars are MACHO 101.20909.1342, 110.22316.115 and 403.47909.1408. Right: Three of the 1028 standard MACHO bulge light curves. From top to bottom these stars are MACHO 104.20388.631, 104.21164.904 and 109.20114.714. The $V$-band magnitudes are plotted as function of phase using the periods from the MACHO database. The white lines represent the 8 th order Fourier fit to the data. The error bars are omitted for clarity. 


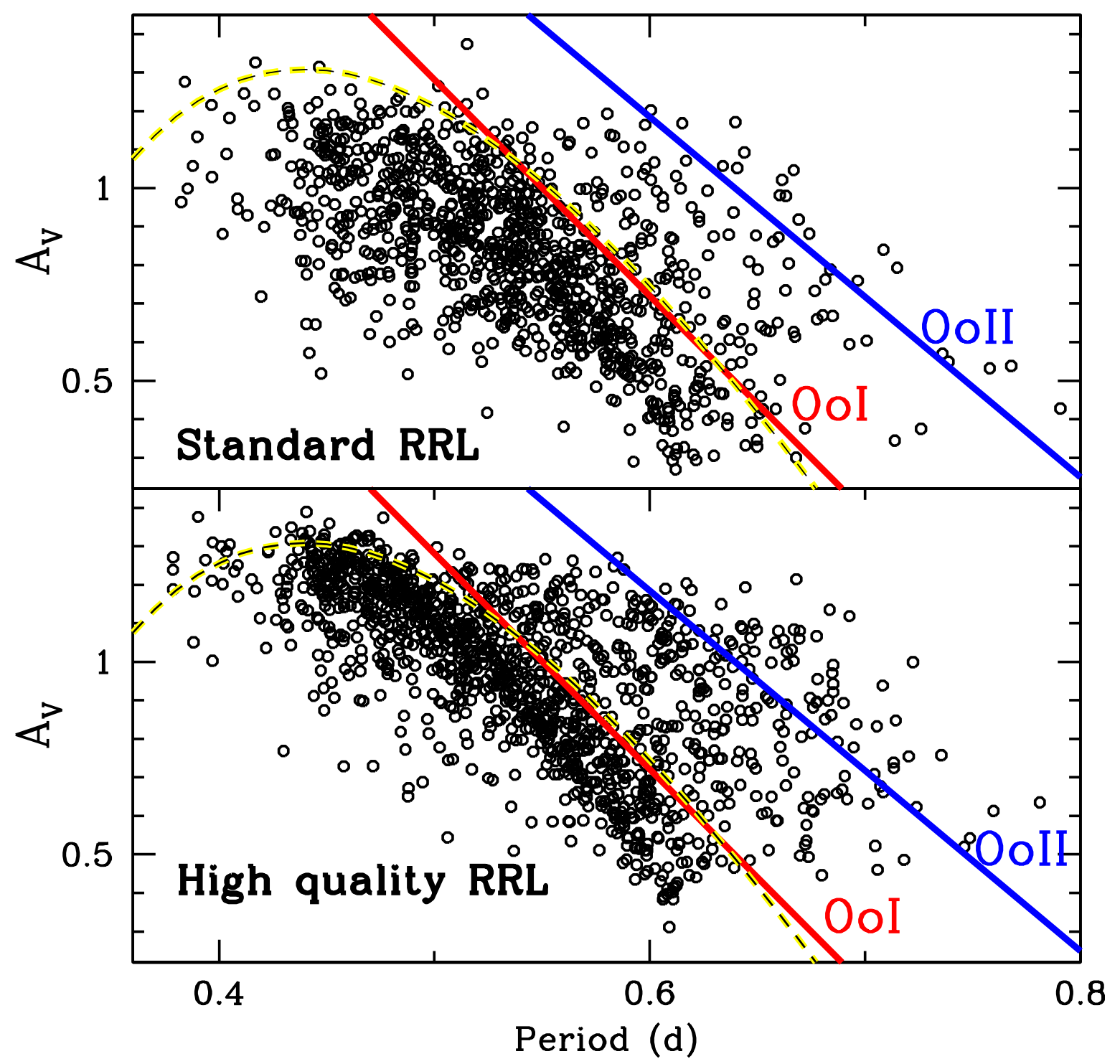

Fig. 2.- Top: Period-amplitude relation for the 1028 standard MACHO Bulge RR0 Lyrae variables with "normal" light curves. Bottom: Same as top panel but with the 1323 stars with high quality bulge light curves. The straight lines were derived by Clement \& Shelton (1999) from a leastsquares fit to the principal sequence of regular RR0 Lyrae stars in the OoI cluster M3 and OoII cluster M9. The dashed line is a quadratic $\mathrm{PA}_{V}$ relation from Cacciari et al. (2005) derived from M3 RR0 Lyrae variables. 


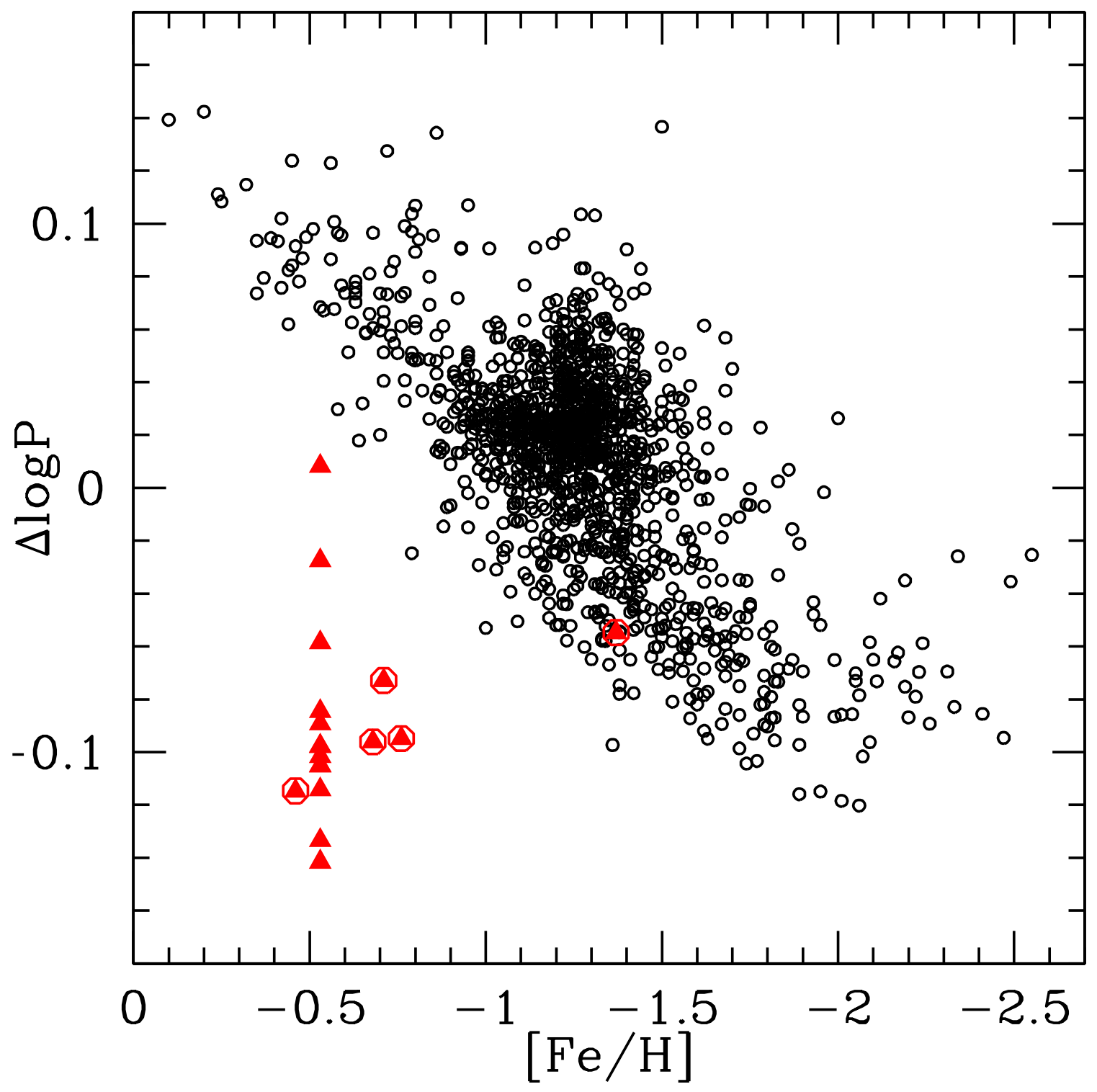

Fig. 3.- $\Delta \log \mathrm{P}$ as a function of $[\mathrm{Fe} / \mathrm{H}]$ for the Bulge field RR0 Lyrae stars with high quality light curves. The red triangles represent RR Lyrae stars in the metal-rich globular cluster NGC 6441with photometry from Pritzl et al. (2003). The red circled triangles represent those stars with spectroscopic metallicities from Clementini et al. (2005). 


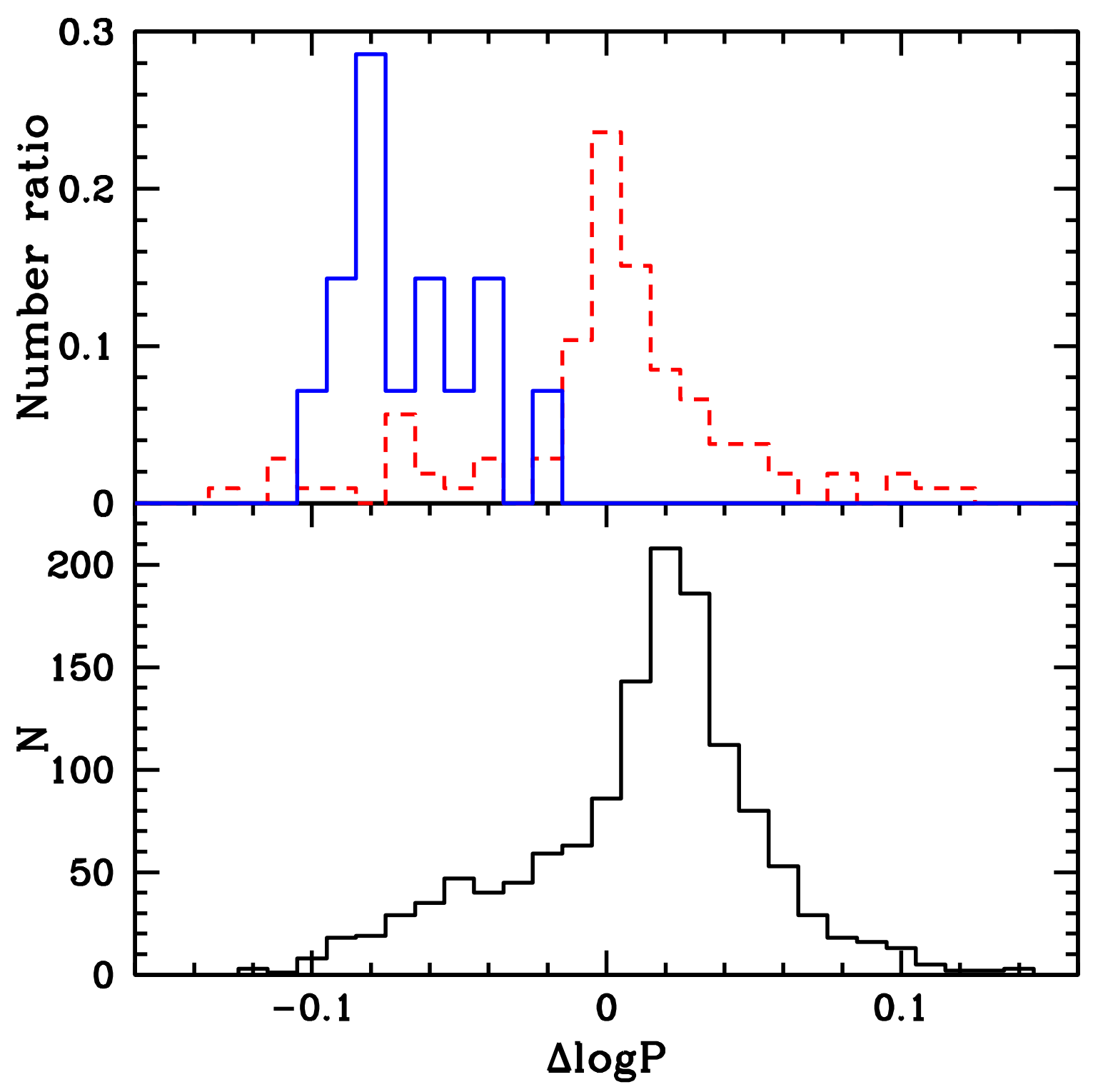

Fig. 4.- Bottom: The histogram of $\Delta \log \mathrm{P}$ from the high quality light curve RR Lyrae stars with respect to the OoI cluster M3. Top: The histogram of RR0 Lyrae stars in the OoI globular clusters M3 and M5 (dashed), and the OoII clusters M2 and M9 (solid). 


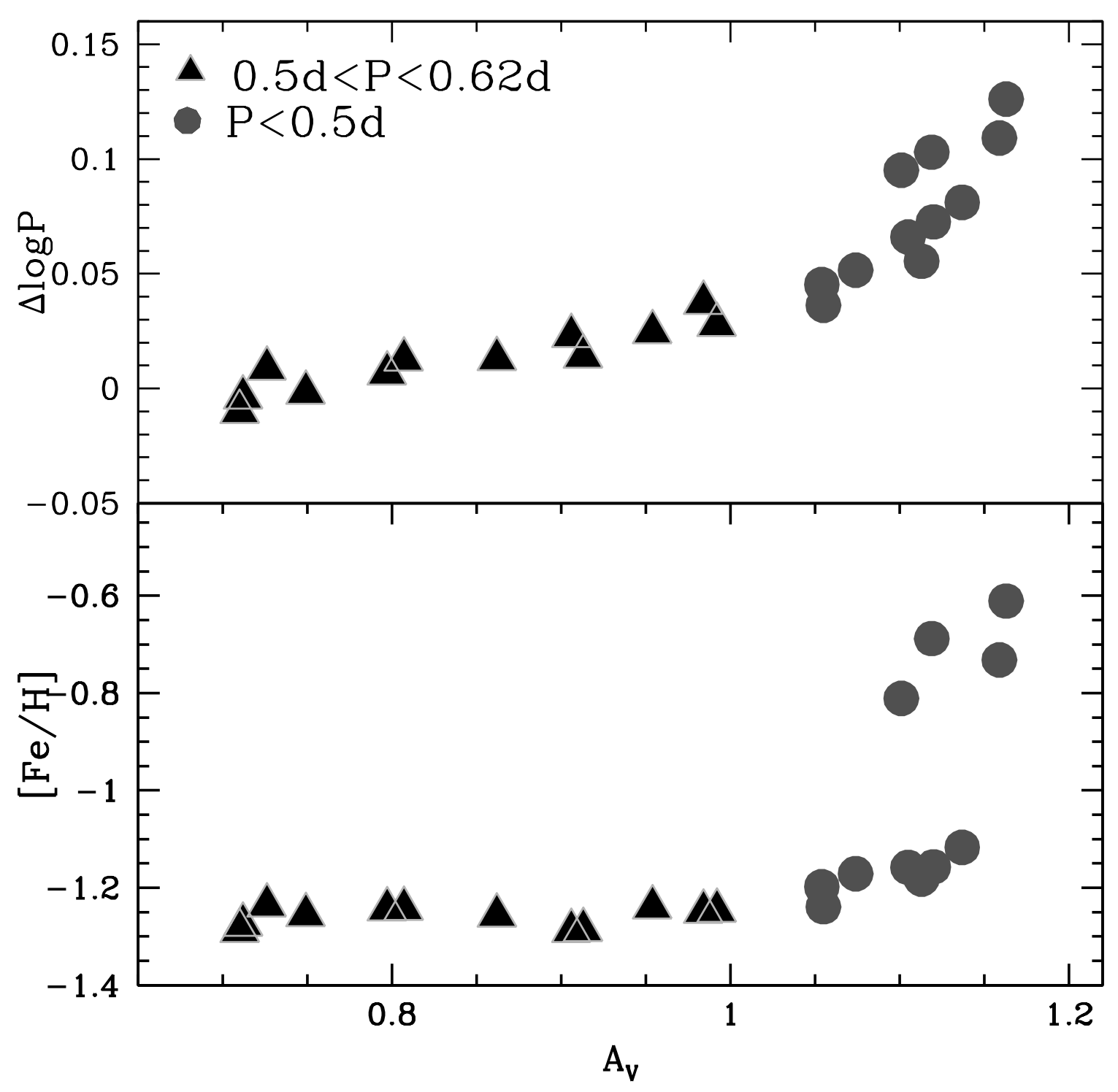

Fig. 5.- The Bulge RR0 Lyrae stars are binned in 0.01 day bins and the average $A_{V}$ in each period bin is plotted as a function of $[\mathrm{Fe} / \mathrm{H}]$ (bottom) and $\Delta \log \mathrm{P}$ (top). 


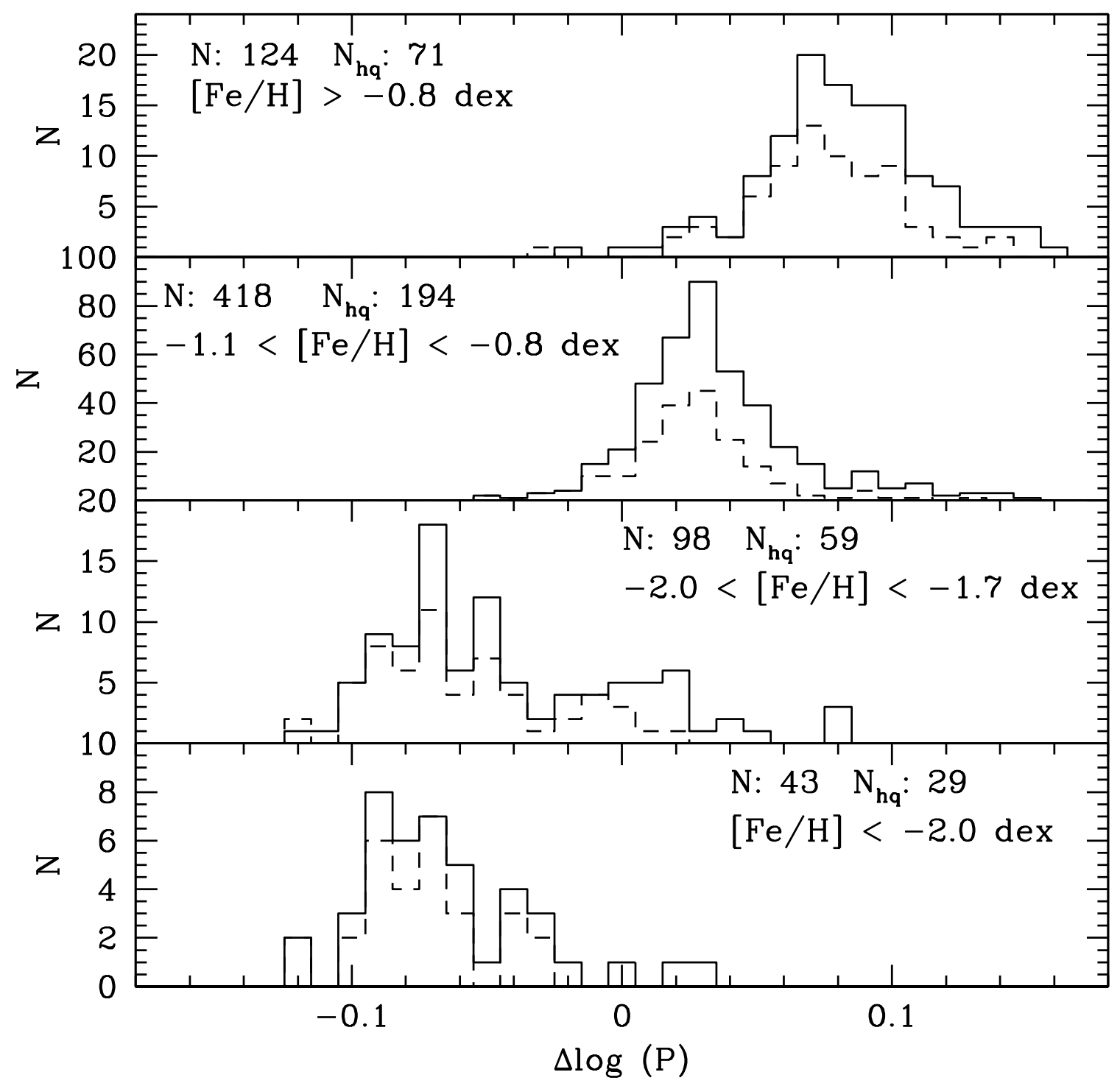

Fig. 6.- Period shift distributions for the Bulge RR0 Lyrae field stars with different metallicity ranges. The dotted lines show the distribution of the RR Lyrae with high quality light curves only. The number of these stars in each metallicity range is given by $\mathrm{N}_{\mathrm{hq}}$, where as the total number of stars in each metallicity range is given by $\mathrm{N}$. 


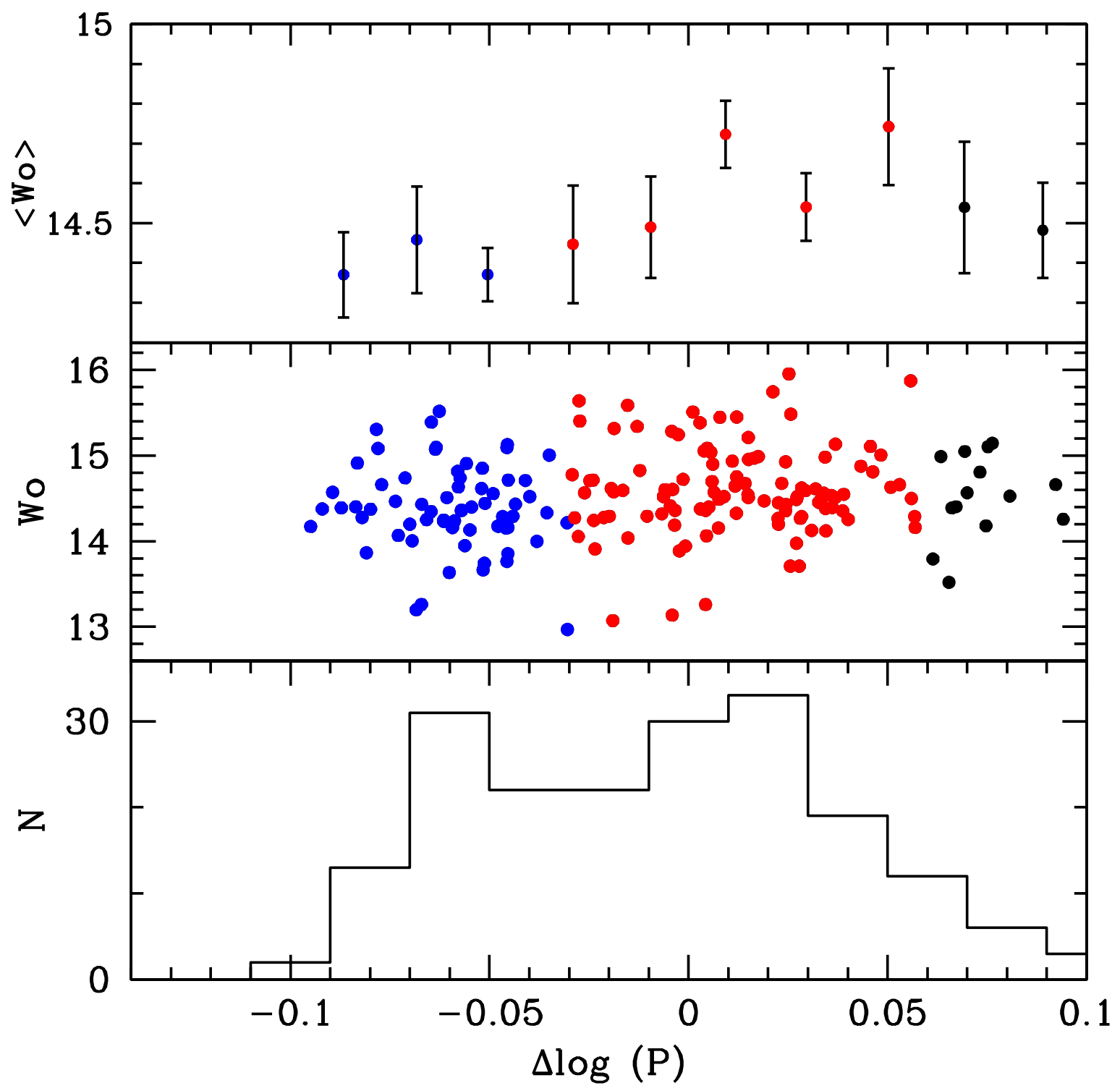

Fig. 7.- Comparison of $\Delta \log \mathrm{P}$ vs. the $\mathrm{W}_{0}$ for $\mathrm{RR} 0$ Lyrae stars with similar $[\mathrm{Fe} / \mathrm{H}]$ values. bottom: Histogram of the distribution of the RR0 Lyrae stars with $-1.7<[\mathrm{Fe} / \mathrm{H}]<-1.5$ dex. middle: Plot of the individual $\Delta \log \mathrm{P}$ values as a function of $W_{0}$. top: Here the RR0 Lyrae stars are binned in $0.02 \Delta \log \mathrm{P}$ bins and plotted against average $W_{0}$. 


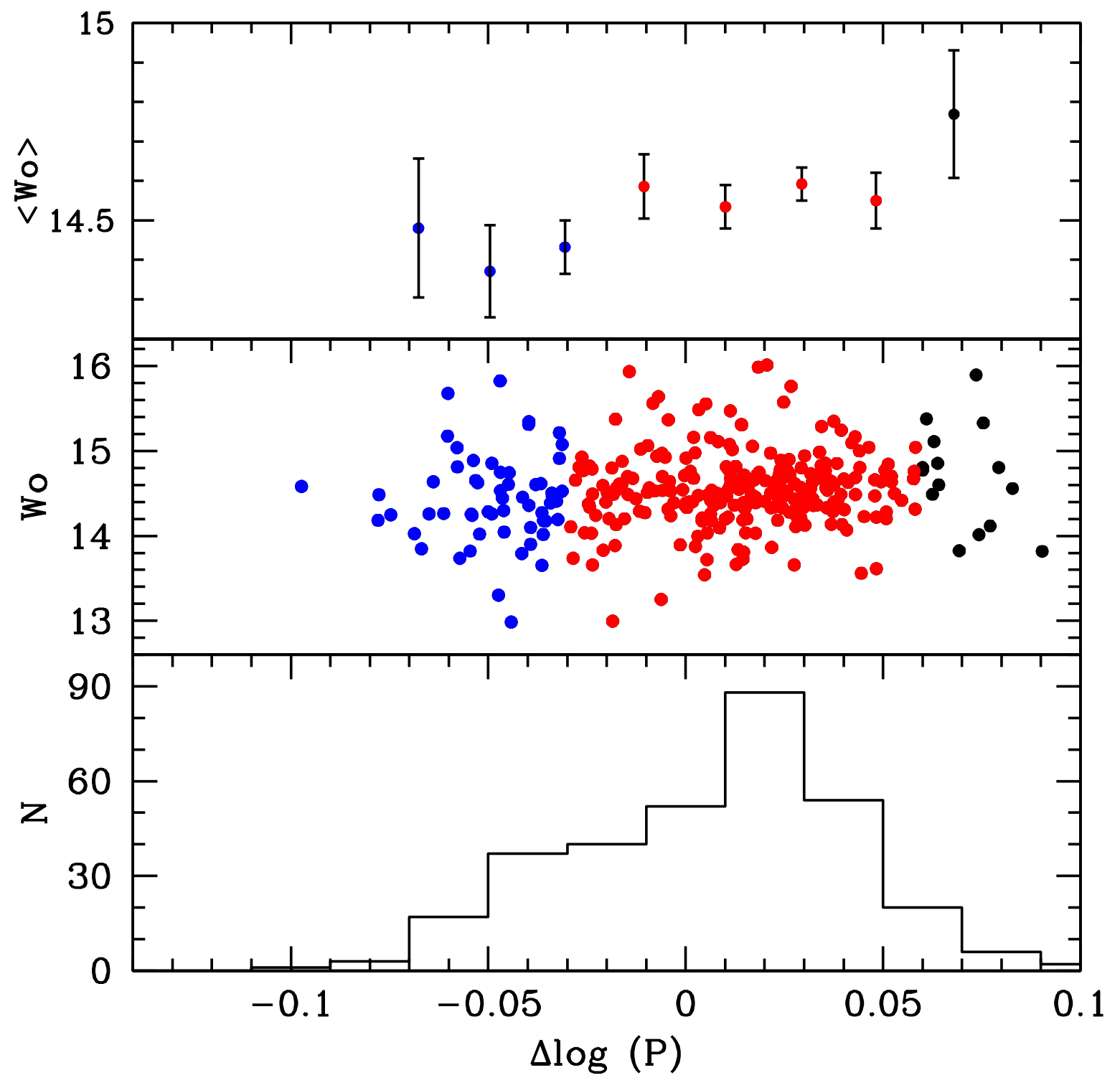

Fig. 8. - Same as Figure [, but for the metallicity distribution $-1.5<[\mathrm{Fe} / \mathrm{H}]<-1.3$ dex. 

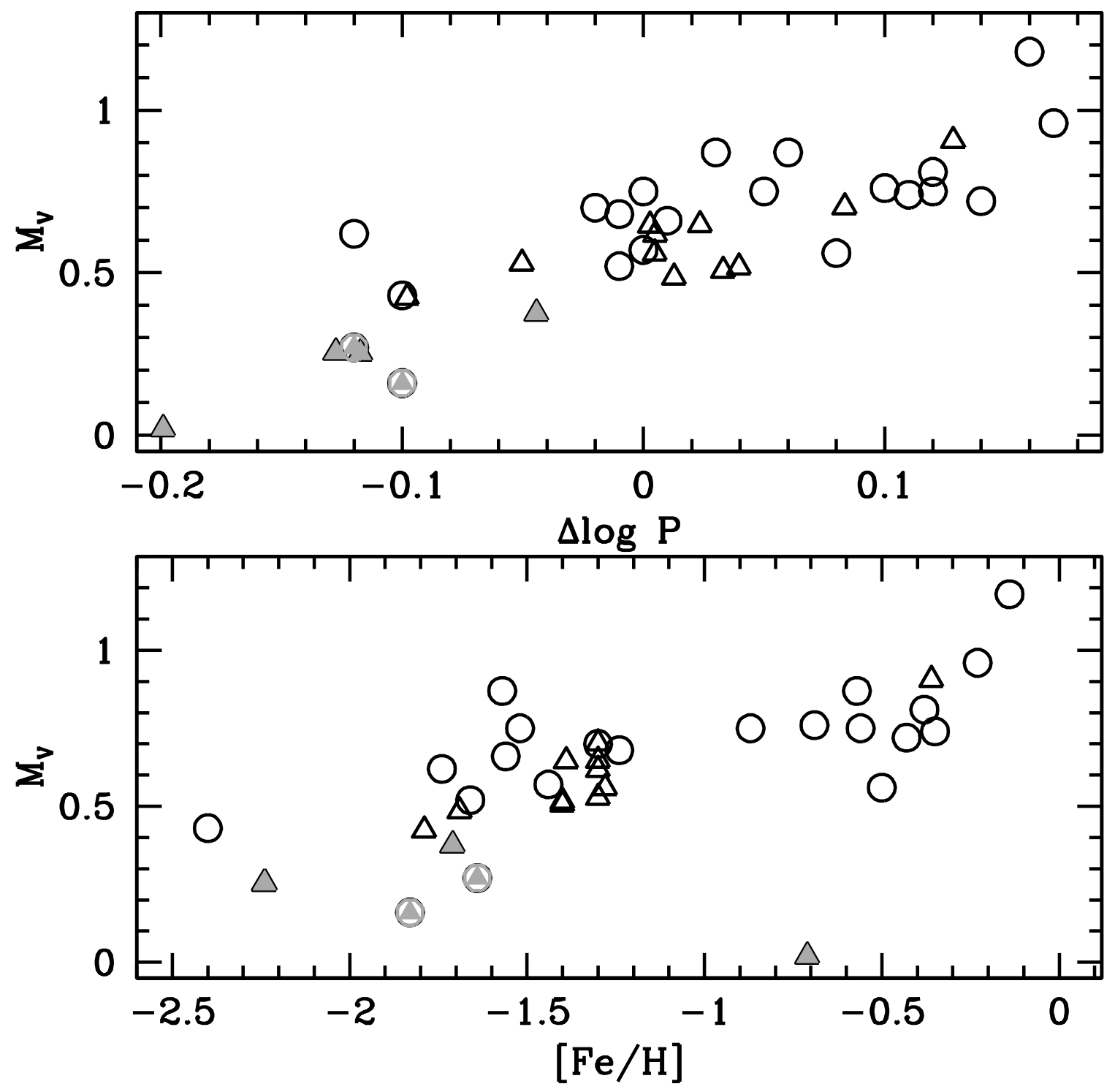

Fig. 9.- A plot of local field RR0 Lyrae stars with $M_{V}^{R R}$ values derived from a Baade-Wesselink analysis, as a function of $[\mathrm{Fe} / \mathrm{H}]$ and $\Delta \log \mathrm{P}$. The open circles indicate those stars with a BaadeWesselink $\mathrm{M}_{V}^{\mathrm{RR}}$ from Kovács (2003) and the open triangles indicate those stars with an $\mathrm{M}_{\mathrm{V}}^{\mathrm{RR}}$ from Bono et al. (2003). The filled triangles highlight those stars with the brightest absolute magnitudes, and seem to be the most discrepant in the $\mathrm{M}_{\mathrm{V}}^{\mathrm{RR}}-[\mathrm{Fe} / \mathrm{H}]$ plot. 